\title{
Chetaev versus vakonomic prescriptions in constrained field theories with parametrized variational calculus
}

\author{
Enrico Bibbona ${ }^{\text {a) }}$ \\ Dipartimento di Matematica, Università degli Studi di Torino, Torino 10123, Italy \\ Lorenzo Fatibene \\ Dipartimento di Matematica, Università degli Studi di Torino, Torino 10123, Italy \\ and INFN, Sezione di Torino, Iniziativa Specifica NA12, 10125 Torino, Italy \\ Mauro Francaviglia \\ Dipartimento di Matematica, Università degli Studi di Torino, Torino 10123, Italy; \\ INFN, Sezione di Torino, Iniziativa Specifica NA12, 10125 Torino, Italy; \\ and ESG, Università della Calabria, 87036 Arcavacata di Rende (CS), Italy
}

(Received 31 August 2006; accepted 22 January 2007; published online 29 March 2007)

\begin{abstract}
Starting from a characterization of admissible Chetaev and vakonomic variations in a field theory with constraints we show how the so called parametrized variational calculus can help to derive the vakonomic and the nonholonomic field equations. We present an example in field theory where the nonholonomic method proved to be unphysical. () 2007 American Institute of Physics. [DOI: 10.1063/1.2709848]
\end{abstract}

\section{INTRODUCTION}

At least two different procedures to obtain the field equations for a mechanical problem with nonintegrable constraints on the velocities have been developed. They are, respectively, called the vakonomic and the nonholonomic method and are both based on variational principles where a suitable restriction on the set of admissible variations is imposed. In the vakonomic (vak) setting the restriction arises from geometric considerations, while in the nonholonomic $(\mathrm{NH})$ case it is derived from d'Alembert principle (see Ref. 10 for a comparison in a common variational framework).

The question of which one of the two methods produces equations, the solutions of which can be physically observed, has been extensively studied and it seems (see Ref. 14) that, at least for a very large class of mechanical constraints, the nonholonomic procedure works better. Nevertheless the vakonomic scheme proved to give interesting results in other frameworks, such as optimal control theory (see, for example, Ref. 4).

In field theories, however, the situation is much less clear: both procedures have been generalized to provide field equations and Nöther currents in some cases (see Refs. 15, 7, and 2 for vak and Refs. 3, 18, and 13 for NH), but it is still no evident which one should be better applied in concrete cases. Moreover no fundamental reason justifies the NH method since d'Alembert principle cannot be formulated.

Here we aim at contributing to this debate by reformulating both methods in terms of parametrized variational calculus: the use of a parametrization sometimes helps to find field equations without any need for additional variables such as Lagrange multipliers.

We also provide a few examples. In particular, we find that if we interpret matter conservation as a nonintegrable constraint in relativistic hydrodynamics, the nonholonomic methods give nonphysical results (every section satisfying the constraint is a solution), while the vakonomic method

\footnotetext{
a)Electronic mail: enrico.bibbona@unito.it
} 
can be successfully implemented. In our knowledge this is the first example of a field theory in which one of the two methods has to be rejected, and surprisingly enough it is exactly the one that works in mechanics.

\section{CONSTRAINED FIELD THEORIES}

Let $C \rightarrow M$ be the configuration bundle whose (global) sections $\Gamma(C)$ represent the fields (by abuse of language we will often denote bundles with the same label as their total spaces). Let moreover $\left(x^{\mu}, y^{i}\right)$ be a fibered coordinate system on $C$.

Definition 1: A first order Lagrangian on the configuration bundle $C \rightarrow M(m=\operatorname{dim} M)$ is $a$ fibered morphism

$$
L: J^{1} C \rightarrow \wedge^{m} T^{*} M
$$

In local coordinates it can be represented as $L\left(x^{\mu}, y^{i}, y_{\mu}^{i}\right) \mathrm{d} s$, where $\mathrm{d} s$ denotes the standard local volume form induced on $M$ by the coordinates $x^{\mu}$.

Definition 2: Let $C \rightarrow M$ be the configuration bundle. A constraint of first order with codimension $r$ is a submanifold $S \subset J^{1} C$ of codimension $r$ that projects onto the whole of $C$.

Henceforth the constraint can be expressed by a set of $r$ independent first order differential equations $\Phi^{(\alpha)}\left(x^{\mu}, y^{i}, y_{\mu}^{i}\right)=0$.

Definition 3: A configuration $\sigma \in \Gamma(C)$ is said to be admissible with respect to $S$ if its first jet prolongation lies in $S$. The space of admissible configurations with respect to $S$ is

$$
\Gamma_{S}(C)=\left\{\sigma \in \Gamma(C) / \operatorname{Im}\left(j^{1} \sigma\right) \in S\right\}
$$

Definition 4: The set $\{C, L, S\}$, where $C \longrightarrow M$ is a configuration bundle, $L$ is a Lagrangian on $C$, and $S$ is a constraint, is called a constrained variational problem.

\section{A. Vak criticality}

Vak criticality was introduced in mechanics (see Ref. 1) as criticality with respect to variations that infinitesimally fulfill the constraint. Here we give a generalization to constrained field theories.

\section{cpt}

Definition 5: Given a compact submanifold $D \subset M$, a vak-admissible variation (at first order) of an admissible configuration $\sigma \in \Gamma_{S}(C)$ is a smooth one parameter family of sections $\left\{\sigma_{t}\right\}_{t \in]-1,1[} \subset \Gamma\left(\pi^{-1} D\right)$ such that

1. $\sigma_{0}=\left.\sigma\right|_{D}$,

2. $\forall t \in]-1,1\left[,\left.\sigma_{t}\right|_{\partial D}=\left.\sigma\right|_{\partial D}\right.$, and

3. $\operatorname{Im}\left(\left.(\mathrm{d} / \mathrm{d} t) j^{1} \sigma_{t}\right|_{t=0}\right) \in J^{1} V C \cap T S$.

In order to check condition 3 , one has to verify that the vertical vector field $W=\left.(\mathrm{d} / \mathrm{d} t) j^{1} \sigma_{t}\right|_{t=0}$ satisfies the following condition:

$$
\frac{\partial \Phi^{(\alpha)}}{\partial y^{i}} W^{i}+\frac{\partial \Phi^{(\alpha)}}{\partial y_{\mu}^{i}} \mathrm{~d}_{\mu} W^{i}=0,
$$

where $W^{i}$ are the components of $W$ along the natural basis $\partial_{i}=\partial / \partial y^{i}$ and the operator $\mathrm{d}_{\mu}$ stands for $\partial_{\mu}+\partial_{\mu} y^{i} \partial_{i}$ and it realizes formally the total derivative with respect to $x^{\mu}$.

Definition 6: We say that an admissible section $\sigma \in \Gamma_{S}(C)$ is vakonomically critical (or vak critical) for the variational problem $\{C, L, S\}$ if $\forall D \subset M$ for any vak-admissible variation $\left\{\sigma_{t}\right\}_{t \in]-1,1[} \in \Gamma\left(\pi^{-1} D\right)$, we have 


$$
\left.\frac{\mathrm{d}}{\mathrm{d} t} \int_{D} L \circ j^{1} \sigma_{t}\right|_{t=0}=0 .
$$

An equivalent infinitesimal condition is the following:

$$
\begin{gathered}
\qquad D \subset M, \quad \forall W \in V C \quad \text { such that } \operatorname{Im}\left[j^{1}(W \circ \sigma)\right] \in T S \text { and }\left.W\right|_{\partial D}=0, \\
\int_{D}\left[\frac{\partial L}{\partial y^{a}} W^{a}+\frac{\partial L}{\partial y_{\mu}^{a}} \mathrm{~d}_{\mu} W^{a}\right] \mathrm{d} s=0 .
\end{gathered}
$$

\section{B. Chetaev criticality}

The classical strategy to get the equation of motion for mechanical systems with constraints is to consider the Lagrangian of the free problem and to equate its Euler-Lagrange operator to the (external) reaction forces due to the constraint. The motivation for this procedure is d'Alembert principle: the work done by reaction forces on any "virtual displacement" has to be zero.

In the holonomic case virtual displacement are infinitesimal displacements allowed by the constraint at a fixed time. In the integrable nonholonomic case (see the next subsection for a rigorous definition and details) the same idea suggests to call virtual displacement a vector field $X \in \Gamma(T M)$ that verifies the conditions $\left[\partial \Phi^{(A)} / \partial \dot{q}^{i}\right] X^{i}=0$.

In the general nonholonomic case one can define a virtual displacement to be a vector field $X$ on $M$ that satisfies the formally analog conditions

$$
\frac{\partial \Phi^{(A)}}{\partial \dot{q}^{i}} X^{i}=0
$$

usually named after Chetaev. The physical interpretation as infinitesimal motions allowed by the constraint is now completely lost, but anyway this is what is usually asked for in nonholonomic mechanics [see, for example, the very classical reference (Ref. 5 or Refs. 4 and 1) for a more recent exposition and interpretation].

Despite the lack of a clear motivation, an experiment (see Ref. 14) proved that in real mechanical systems where the two procedures give different equations the observed trajectories are solutions of the equations of motion derived according to Chetaev rule and not of those arising in the vakonomic framework. In Refs. 3, 18, and 13 the Chetaev framework has been generalized to field theories and here we describe a concept of criticality that follows this scheme.

Definition 7: Given a compact submanifold $D \subset M$ a Chetaev-admissible variation of an admissible configuration $\sigma \in \Gamma_{S}(C)$ is a smooth one parameter family of sections $\left\{\sigma_{t}\right\}_{t \in]-1,1[} \subset \Gamma\left(\pi^{-1} D\right)$ such that

1. $\sigma_{0}=\left.\sigma\right|_{D}$,

2. $\forall t \in]-1,1\left[,\left.\sigma_{t}\right|_{\partial D}=\left.\sigma\right|_{\partial D}\right.$, and

3. the vertical vector field $\left.(\mathrm{d} / \mathrm{d} t) \sigma_{t}\right|_{t=0}=W$ on $\sigma$ with coordinate expression $W=W^{i} \partial_{i}$ is such that $\left[\partial \Phi^{(\alpha)} / \partial y_{\mu}^{i}\right] W^{i}=0$.

Definition 8: We say that an admissible section $\sigma \in \Gamma_{S}(C)$ is Chetaev critical for the varia$\mathrm{cpt}$

tional problem $\{C, L, S\}$ if $\forall D \subset M$ for any Chetaev-admissible variation $\left\{\sigma_{t}\right\}_{t \in]-1,1[} \in \Gamma\left(\pi^{-1} D\right)$ we have 


$$
\left.\frac{\mathrm{d}}{\mathrm{d} t} \int_{D} L \circ j^{1} \sigma_{t}\right|_{t=0}=0
$$

An equivalent infinitesimal condition is the following:

$$
\begin{gathered}
\forall D \subset M, \quad \forall W \in V C \quad \text { such that } \frac{\partial \Phi^{(\alpha)}}{\partial y_{\mu}^{i}} W^{i}=0 \text { and }\left.W\right|_{\partial D}=0, \\
\int_{D}\left[\frac{\partial L}{\partial y^{a}} W^{a}+\frac{\partial L}{\partial y_{\mu}^{a}} \mathrm{~d}_{\mu} W^{a}\right] \mathrm{d} s=0 .
\end{gathered}
$$

\section{Integrable constraints}

Definition 9: Given a vertical fibered morphism $f=(i d, \Phi)$ between two bundles $B$ and $D$ over the same base $M$ we call first order jet prolongation of $f$ the unique fibered morphism $j^{1} f$ $=\left(i d, j^{1} \Phi\right)$ that makes the following diagram commutative:

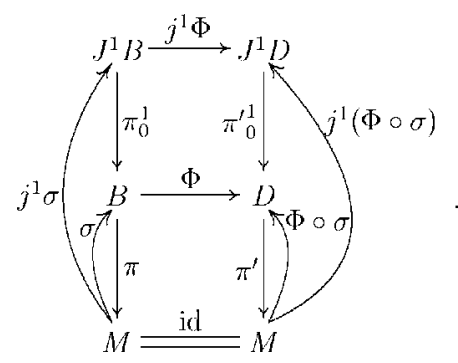

Let $\left(x^{\mu}, y^{i}\right)$ be coordinates on $B$ and $\left(x^{\mu}, y^{i}\right)$ coordinates on $D$. Let moreover $z^{A}\left(x^{\mu}, y^{i}\right)$ be the coordinate expression of $f$. Its prolongation $j^{1} f$ has coordinate expression

$$
z_{\mu}^{A}=\mathrm{d}_{\mu} z^{A}=\frac{\partial z^{A}}{\partial x^{\mu}}+y_{\mu}^{i} \frac{\partial z^{A}}{\partial y^{i}},
$$

where the operator $\mathrm{d}_{\mu}$, called formal derivative, realizes formally the total derivative with respect to $x^{\mu}$.

Theorem 1: Let $S \in J^{1} C$ be a set of integrable constraints linear in the derivatives, locally expressed as the zero set of the prolongation $\Phi_{\mu}^{(\alpha)}$ of a morphism $f: C \rightarrow E$ ( $E$ is a vector bundle), i.e., in the form $\Phi_{\mu}^{(\alpha)}=\mathrm{d}_{\mu} f^{(\alpha)}(x, y)=0$. With respect to $S$ any Chetaev-admissible variation is also vak admissible and vice versa.

Proof: The condition for a vertical vector field $W=\left.(\mathrm{d} / \mathrm{d} t) \sigma_{t}\right|_{t=0}$ to be relative to a Chetaev admissible variation is that

$$
\frac{\partial \Phi_{\mu}^{(\alpha)}}{\partial y_{\nu}^{i}} W^{i}=\delta_{\mu}^{\nu} \frac{\partial f^{(\alpha)}(x, y)}{\partial y^{i}} W^{i}=0 \Leftrightarrow \frac{\partial f^{(\alpha)}(x, y)}{\partial y^{i}} W^{i}=0 .
$$

On the other hand, for vak admissibility the following condition is needed:

$$
\frac{\partial \Phi_{\mu}^{(\alpha)}}{\partial y^{i}} W^{i}+\frac{\partial \Phi_{\mu}^{(\alpha)}}{\partial y_{\nu}^{i}} \mathrm{~d}_{\nu} W^{i}=0 \Leftrightarrow \mathrm{d}_{\mu} \frac{\partial f^{(\alpha)}}{\partial y^{i}} W^{i}+\delta_{\mu}^{\nu} \frac{\partial f^{(\alpha)}}{\partial y^{i}} \mathrm{~d}_{\nu} W^{i}
$$

and this is equivalent to 


$$
\mathrm{d}_{\mu}\left(\frac{\partial f^{(\alpha)}}{\partial y^{i}} W^{i}\right)=0
$$

Now obviously Eq. (3) implies Eq. (4). Conversely, the validity of Eq. (4) together with the vanishing of $W$ at the boundary implies Eq. (3).

Corollary 1: For any constrained variational principle $\{C, L, S\}$ with integrable constraint $S$ every vakonomically critical section is Chetaev critical and vice versa.

\section{VARIATIONAL CALCULUS WITH PARAMETRIZED VARIATIONS}

For the sake of mathematical rigor let us introduce some technical details on the different bundle structures involved in the following definitions. The reader more interested in applications could safely skip to the coordinate version of the definition of a parametrization of the constrained variations.

Let $C \rightarrow M$ a bundle and $E \rightarrow C$ a vector bundle with coordinates $\left(x^{\mu}, y^{a}\right)$ on the base $C$ and $\varepsilon^{A}$ on the fiber. One can also regard it as a bundle with base $M$ with the composite projection: in the latter sense the coordinates on the base are $x^{\mu}$, and those on the fiber are $\left(y^{a}, \varepsilon^{A}\right)$. The bundle $E$ $\rightarrow M$ has prolongation $J^{1} E \rightarrow M$. Appropriate coordinates on $J^{1} E \rightarrow M$ are $\left(x^{\mu}, y^{a}, \varepsilon^{A}, y_{\mu}^{a}, \varepsilon_{\mu}^{A}\right)$. One can consider the intermediate projection $J^{1} E \rightarrow J^{1} C$ that locally reads as $\left(x^{\mu}, y^{a}, y_{\mu}^{a}, \varepsilon^{A}, \varepsilon_{\mu}^{A}\right) \mapsto\left(x^{\mu}, y^{a}, y_{\mu}^{a}\right)$. It gives rise to the vector bundle $J^{1} E \rightarrow J^{1} C$. The situation is summarized by the following diagram:

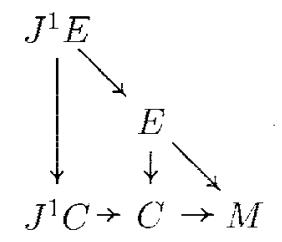

In what follows by $J^{1} E$ we will always denote the bundle $J^{1} E \rightarrow J^{1} C$, moreover when required bundles are meant as pulled back on the appropriate base (e.g., $V C$ or $\Lambda^{m} T^{*} M$ are often regarded as bundles on $J^{1} C$ meaning a pullback along the appropriate projection). For the sake of readability of the formulas we will omit the pullback notation.

Definition 10: A parametrization of order 1 and rank 1 of the set of constrained variations is a couple $(E, \mathrm{P})$, where $E$ is a vector bundle $E \rightarrow C$, while $\mathrm{P}$ is a fibered morphism (section)

$$
\mathrm{P}: J^{1} C \rightarrow\left(J^{1} E\right)^{*} \otimes_{J^{1} C} V C .
$$

If $\left(x^{\mu}, y^{a}, \varepsilon^{A}\right)$ are local fibered coordinates on $E$ and $\left\{\partial_{a}\right\}$ is the induced fiberwise natural basis of $V C$, a parametrization of order 1 and rank 1 is thence a couple of coefficients $\left(p_{A}^{a}, p_{A}^{a}{ }^{\mu}\right)$ functions of $\left(x^{\nu}, y^{b}(x), \partial_{\nu} y^{b}(x)\right)$ that under the vector bundle coordinate change

$$
\begin{gathered}
x^{\mu^{\prime}}=x^{\mu^{\prime}}\left(x^{\mu}\right), \\
y^{a^{\prime}}=y^{a^{\prime}}\left(x^{\mu}, y^{a}\right), \\
\varepsilon^{A^{\prime}}=M_{A}^{A^{\prime}}\left(x^{\mu}, y^{a}\right) \varepsilon^{A}
\end{gathered}
$$

transform in such a way that for any section $y^{a}(x)$ of $C$ and any section $\varepsilon^{A}(x, y)$ of $E$ the vector field

$$
W=\left[p_{A}^{a} \varepsilon^{A}+p_{A}^{a}{ }^{\mu} d_{\mu} \varepsilon^{A}\right] \partial_{a}
$$

transforms as a section of $V C$.

The explicit transformation rules required are the following: 


$$
\begin{gathered}
p_{A}^{a}=J_{a^{\prime}}^{a}\left(p_{A^{\prime}}^{a^{\prime}} M_{A}^{A^{\prime}}+p_{A^{\prime}}^{a^{\prime} \mu^{\prime}} J_{\mu^{\prime}}^{\mu} \mathrm{d}_{\mu} M_{A}^{A^{\prime}}\right), \\
p_{A}^{a}{ }^{\mu}=J_{a^{\prime}}^{a} J_{\mu^{\prime}}^{\mu} \mathrm{d}_{\mu} M_{A}^{A^{\prime}} p_{A^{\prime}}^{a^{\prime} \mu^{\prime}},
\end{gathered}
$$

where $J_{a^{\prime}}^{a}=\partial y^{a} / \partial y^{a^{\prime}}$ and $J_{\mu^{\prime}}^{\mu}=\partial x^{\mu} / \partial x^{\mu^{\prime}}$. are Jacobean matrices.

The particular choice of the coefficients $\left(p_{A}^{a}, p_{A}^{a}{ }^{\mu}\right)$ selects the admissible variations according to the following definition.

Definition 11: Given a compact submanifold $D \subset M$ a P-admissible variation of a configuration $\sigma \in \Gamma(C)$ on $D$ is a smooth one parameter family of sections $\left\{\sigma_{t}\right\}_{t \in]-1,1[} \subset \Gamma\left(\pi^{-1} D\right)$ such that

1. $\sigma_{0}=\left.\sigma\right|_{D}$,

2. $\forall t \in]-1,1\left[,\left.\quad \sigma_{t}\right|_{\partial D}=\left.\sigma\right|_{\partial D}\right.$, and

3. there exists a section $\varepsilon \in \Gamma(E)$ such that $\left.(\mathrm{d} / \mathrm{d} t) j^{1} \sigma_{t}\right|_{t=0}=\left\langle\mathbb{P} \mid j^{1} \varepsilon\right\rangle \circ j^{1} \sigma$ and $\left.j^{1}\left(\varepsilon^{\circ} \sigma\right)\right|_{\partial D}=0$.

Definition 12: The set $\{C, L, \mathbb{P}\}$, where $C \rightarrow M$ is a configuration bundle, $L$ is a Lagrangian on $C$, and $\mathbb{P}$ is a parametrization of the set of constrained variations, is called a parametrized variational problem.

Definition 13: We define $P$ critical for the parametrized variational problem $\{C, L, P\}$ those sections of $C$ for which, for any compact $D \subset M$ and for any P-admissible variation $\left\{\sigma_{t}\right\}$ defined on $D$, one has

$$
\left.\frac{\mathrm{d}}{\mathrm{d} t} \int_{D} L \circ j^{1} \sigma_{t}\right|_{t=0}=0
$$

Accordingly, if we use the trivial parametrization $\mathrm{P}: C \rightarrow V C^{*} \otimes_{C} V C$ that to any point $p \in C$ associates the identity matrix of $V_{p} C$ then the third condition becomes empty and we recover free variational calculus [we can reinterpret $\mathrm{P}$ as a map $J^{1} C \rightarrow\left(J^{1} V C\right)^{*} \otimes_{C} V C$ that do not depend on the derivatives in order to match the definition of parametrization of order 1 and rank 1].

For an ordinary variational problem with Lagrangian $L=L \mathrm{~d} s$ criticality of a section of $C$ is equivalent, in local fibered coordinates $\left(x^{\mu}, y^{a}\right)$, to the fact that for any compact $D \subset M$ and for any $W \in \Gamma(V C)$ such that $\left.W \circ \sigma\right|_{\partial D}=0$ one has

$$
\int_{D}\left[\frac{\partial L}{\partial y^{i}} W^{i}(x, y(x))+\frac{\partial L}{\partial y_{\mu}^{i}} \mathrm{~d}_{\mu} W^{i}(x, y(x))\right] \mathrm{d} s=0 .
$$

Explicit calculations (see Ref. 6) show that the above local coordinate expressions glue together with the neighboring, giving rise to the following global one:

$$
\begin{gathered}
\forall D \subset M, \quad \forall W \in V C \quad \text { such that }\left.(W \circ \sigma)\right|_{\partial D}=0, \\
\int_{D}\left\langle\delta L \mid j^{1} W\right\rangle \circ j^{1} \sigma=0,
\end{gathered}
$$

where $\delta L$ is a fibered morphism

$$
\delta L: J^{1} C \rightarrow\left(J^{1} V C\right)^{*} \otimes_{J^{1}} C^{m} T^{*} M
$$

and the symbol $\langle\mid\rangle$ recalls the pairing between sections of $\left(J^{1} V C\right)^{*}$ and sections of $J^{1} V C$.

To define criticality for first order parametrized variational problems we have to restrict variations to those $W \in \Gamma(V C)$ in Eq. (6) with $\left.(W \circ \sigma)\right|_{\partial D}=0$ that can be obtained through the parametrization from a section $\varepsilon$ of $E$ satisfying $\left.j^{1}\left(\varepsilon^{\circ} \sigma\right)\right|_{\partial D}=0$. If $\left(x^{\mu}, y^{a}, \varepsilon^{A}\right)$ are local fibered coordinates on $E$ and $\left(p_{A}^{a} \varepsilon^{A}+p_{A}^{a} \mu_{\mu} \varepsilon_{\mu}^{A}\right) \partial_{a}$ is the local representation of $\left\langle\mathbb{P} \mid j^{1} \varepsilon\right\rangle \circ j^{1} \sigma$, criticality 
holds if and only if for any compact $D \subset M$ for any section $\varepsilon$ with coordinate expression $\varepsilon^{A}(x, y)$ such that both $\varepsilon^{A}(x, y(x))=0$ and $d_{\mu} \varepsilon^{A}(x, y(x))=0$ for all $x \in \partial D$, we have

$$
\int_{D}\left[\frac{\partial L}{\partial y^{a}}\left(p_{A}^{a} \varepsilon^{A}+p_{A}^{a} \mu_{\mu} \varepsilon_{\mu}^{A}\right)+\frac{\partial L}{\partial y_{\mu}^{a}} \mathrm{~d}_{\mu}\left(p_{A}^{a} \varepsilon^{A}+p_{A}^{a}{ }^{\nu} \mathrm{d}_{\nu} \varepsilon^{A}\right)\right] \mathrm{d} s=0 .
$$

To set up a characterization of critical sections in terms of a set of differential equations let us introduce the following procedure: let us split the integrand of Eq. (8) into a first summand that factorizes $\varepsilon^{A}$ (without any derivative) plus the total derivative of a second term (a general theorem ensures that this splitting is unique; see Ref. 2). To do this, we integrate by parts the derivatives of $\varepsilon$ in the integrand of Eq. (8). What we get is

$$
\int_{D}\left(\mathbb{E}_{A} \varepsilon^{A}+\mathrm{d}_{\mu}\left(\mathbb{F}_{A}^{\mu} \varepsilon^{A}+\mathbb{F}_{A}^{\mu \nu} \mathrm{d}_{\nu} \varepsilon^{A}\right)\right) \mathrm{d} s=0
$$

with

$$
\begin{gathered}
\mathbb{E}_{A}=\left(\frac{\partial L}{\partial y^{a}}-\mathrm{d}_{\nu} \frac{\partial L}{\partial y_{\nu}^{a}}\right) p_{A}^{a}-\mathrm{d}_{\mu}\left[\left(\frac{\partial L}{\partial y^{a}}-\mathrm{d}_{\nu} \frac{\partial L}{\partial y_{\nu}^{a}}\right) p_{A}^{a} \mu\right] \\
\mathbb{F}_{A}^{\mu}=\left(\frac{\partial L}{\partial y^{a}}-\mathrm{d}_{\nu} \frac{\partial L}{\partial y_{\nu}^{a}}\right) p_{A}^{a} \mu+\frac{\partial L}{\partial y_{\mu}^{a}} p_{A}^{a}, \\
\mathbb{F}_{A}^{\mu \nu}=\frac{\partial L}{\partial y_{\mu}^{a}} p_{A}^{a \nu} .
\end{gathered}
$$

The desired characterization of P-critical sections by means of a set of differential equations is now achieved, in fact, thanks to Stokes's theorem, the vanishing of $j^{1} \varepsilon$ on the boundary and the arbitrariness of $D$ [Eq. (9)] holds if and only if $\sigma$ is a solution of the P-Euler-Lagrange equations $\mathbb{E}_{A}=0$. In Ref. 2 we have shown that the coefficients $\mathbb{E}_{A}, \mathbb{F}_{A}^{\mu}$, and $\mathbb{F}_{A}^{\mu \nu}$ are the components of two global morphisms

$$
\begin{gathered}
\mathbb{E}(L, \mathbb{P}): J^{3} C \rightarrow E^{*} \otimes_{C} \Lambda^{m} T^{*} M, \\
\mathbb{F}(L, \mathbb{P}, \gamma): J^{2} C \rightarrow\left(J^{1} E\right)^{*} \otimes_{J^{1} C} \Lambda^{m} T^{*} M
\end{gathered}
$$

such that the vanishing of the former on a section expresses its criticality, while the latter is linked with the conserved currents that in many cases can be related to the symmetries of the variational problem (the gauge-natural case is developed in detail). In the same paper we also showed that whole procedure can be given a global meaning in terms of variational morphisms and global operations between them. The same has also been done for higher order Lagrangians and for higher rank and higher order parametrizations.

\section{A. Vak-adapted parametrization}

Definition 14: Let $\stackrel{\pi}{\rightarrow} M$ be the configuration bundle. A parametrization

$$
\mathbb{P}_{S}: J^{1} C \rightarrow\left(J^{1} E\right)^{*} \otimes_{J^{1} C} V C
$$

of the set of constrained variations is said to be vak adapted to the constraint $S \subset J^{1} C$ if for all $\varepsilon \in \Gamma(E)$ and $\sigma \in \Gamma_{S}(C)$ the vertical vector field $j^{1}\left(\left\langle\mathbb{P} \mid j^{1} \varepsilon\right\rangle \circ j^{1} \sigma\right)$ has image inTS. 
To prove vak adaptedness to a constraint given by the equations $\Phi^{(\alpha)}=0$ one has to check that the parametrization with coordinate expression (5) automatically implements conditions (1) or, in formulas, that we have

$$
\frac{\partial \Phi^{(\alpha)}}{\partial y^{a}}\left[p_{A}^{a} \varepsilon^{A}+p_{A}^{a} \mu_{\mathrm{d}_{\mu}} \varepsilon^{A}\right]+\frac{\partial \Phi^{(\alpha)}}{\partial y_{\mu}^{a}} \mathrm{~d}_{\mu}\left[p_{A}^{a} \varepsilon^{A}+p_{A}^{a}{ }^{\nu} \mathrm{d}_{\nu} \varepsilon^{A}\right]=0 .
$$

Definition 15: A parametrization $\mathrm{P}_{S}$ vak adapted to a constraint $S$ is said to be vak faithful on $\sigma \in \Gamma_{S}(C)$ to $S$ if for all $W \in V C$ such that both $\operatorname{Im}\left[j^{1}(W \circ \sigma)\right] \in T S$ and $\left.(W \circ \sigma)\right|_{\partial D}=0$ hold, there exists a section $\varepsilon \in \Gamma(E)$ such that $\left\langle\mathbb{P} \mid j^{1} \varepsilon\right\rangle \circ j^{1} \sigma=W$ and $\left.j^{1}\left(\varepsilon^{\circ} \sigma\right)\right|_{\partial D}=0$.

The fundamental problem of the existence of a faithful parameterization that is vakonomically adapted to a constraint $S$ has been studied recently in Ref. 9, where a universal faithful parametrization has been found for any constraint satisfying certain (quite restrictive) conditions. However, we stress that also nonfaithful parametrizations can be useful for some specific tasks (see Sec. IV B 1 and Remark 5).

Proposition 1: Let $\sigma \in \Gamma_{S}(C)$ be a vakonomically critical section for the constrained variational problem $\{C, L, S\}$, then for any adapted parametrization $\mathbb{P}_{S}$ the section $\sigma$ is $\mathbb{P}_{S}$ critical.

Proof: We have

$$
\begin{aligned}
& \sigma \in \Gamma_{S}(C) \text { is vak critical } \\
& \mathbb{1} \\
& \forall D \subset M, \quad \forall \text { adm . var. }\left\{\sigma_{\varepsilon}\right\},\left.\quad \frac{\mathrm{d}}{\mathrm{d} \varepsilon} \int_{D} L \circ j^{1} \sigma_{\varepsilon}\right|_{\varepsilon=0}=0 \\
& \Uparrow \\
& \forall D \subset M, \quad \forall W \in V C \quad \text { such that } \operatorname{Im}\left[j^{1}(W \circ \sigma)\right] \in T S \text { and }\left.\sigma\right|_{\partial D}=0, \\
& \int_{D}\left\langle\delta L \mid j^{1} W\right\rangle \circ j^{1} \sigma=0 \\
& \Downarrow(\mathrm{a}) \\
& \text { cpt } \\
& \forall D \subset M, \quad \forall \varepsilon \in \Gamma(E) \text { such that }\left.j^{2} \varepsilon\right|_{\partial D}=0, \\
& \int_{D}\left\langle\left\langle\delta L \mid j^{1} \mathbb{P}\right\rangle \mid j^{2} \varepsilon\right\rangle \circ j^{3} \sigma=0 \\
& \Uparrow \\
& \mathbb{E} \circ j^{3} \sigma=0 \text {. }
\end{aligned}
$$

In the previous formulae the symbols $\langle\mid\rangle$ recall the pairings between sections of $\left(J^{1} V C\right)^{*}$ and sections of $J^{1} V C$ and between sections of $\left(J^{2} E\right)^{*}$ and sections of $J^{2} E$. Step (a) is not an equivalence since there can be admissible infinitesimal variations vanishing at the boundary with their derivatives up to the desired order that do not come from sections of the bundle of parameters that do vanish on the boundary. The last equivalence holds in force of Stokes's theorem, the vanishing of $j^{1} \varepsilon$ on the boundary and the independence of the generators of $E$.

Corollary 2: Let $\sigma \in \Gamma_{S}(C)$ be an admissible $\mathbb{P}_{S^{-}}$critical section for the constrained variational problem $\{C, L, S\}$ and let also $\mathbb{P}_{S}$ be faithful to $S$ on $\sigma$, then $\sigma$ is vakonomically critical for the constrained variational problem $\{C, L, S\}$.

\section{B. Chetaev-adapted parametrization}

Definition 16: Let $\stackrel{\pi}{\rightarrow} M$ be the configuration bundle. A parametrization

$$
\mathbb{P}_{S}: J^{1} C \rightarrow\left(J^{1} E\right)^{*} \otimes_{J^{1} C} V C
$$




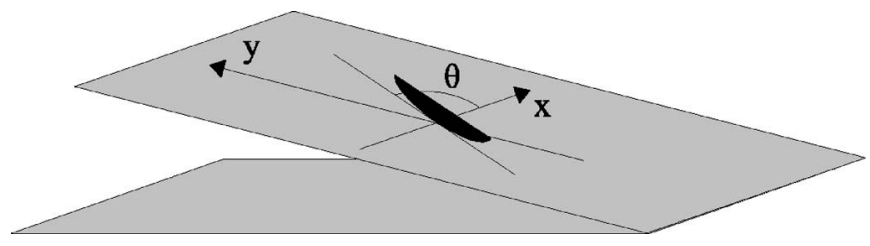

FIG. 1. A skate on an inclined plane.

of the set of constrained variations is said to be Chetaev adapted to the constraint $S \subset J^{1} C$ if for all $\varepsilon \in \Gamma(E)$ and $\sigma \in \Gamma_{S}(C)$ the vertical vector field $\left\langle\mathbb{P} \mid j^{1} \varepsilon\right\rangle \circ j^{1} \sigma$ satisfies conditions 3 of Definition 7.

In coordinates, given expression (5) for the parametrization, such conditions reads as

$$
\frac{\partial \Phi^{(\alpha)}}{\partial y_{\nu}^{a}}\left[p_{A}^{a} \varepsilon^{A}+p_{A}^{a} \mu_{\mathrm{d}_{\mu}} \varepsilon^{A}\right]=0 .
$$

Definition 17: A parametrization $\mathbb{P}_{S}$ Chetaev adapted to a constraint $S$ is said to be Chetaev faithful on $\sigma \in \Gamma_{S}(C)$ to $S$ if for all $W \in V C$ such that both $\partial \Phi^{(\alpha)} / \partial y_{\mu}^{i}(W \circ \sigma)^{i}=0$ and $\left.(W \circ \sigma)\right|_{\partial D}$ $=0$ hold, there exists a section $\varepsilon \in \Gamma(E)$ such that $\left\langle P \mid j^{1} \varepsilon\right\rangle \circ j^{1} \sigma=W$ and $\left.j^{1}\left(\varepsilon^{\circ} \sigma\right)\right|_{\partial D}=0$.

As we did in the previous section we can prove the following proposition.

Proposition 2: Let $\sigma \in \Gamma_{S}(C)$ be a Chetaev critical section for the constrained variational problem $\{C, L, S\}$. For any Chetaev-adapted parametrization $\mathbb{P}_{S}$ the section $\sigma$ is also $\mathbb{P}_{S}$ critical.

Corollary 3: Let $\sigma \in \Gamma_{S}(C)$ be an admissible $\mathbb{P}_{S^{-}}$critical section for the constrained variational problem $\{C, L, S\}$ with respect to a faithful $\mathbb{P}_{S}$, then $\sigma$ is also Chetaev critical.

\section{EXAMPLES}

In literature very few examples of Lagrangian field theories with constraints are present and the question whether the Chetaev or the vakonomic rule produces equations, whose solutions are physically observed, is still open. Let us present here two examples: the first is a classic in mechanics with nonholonomic constraint, while the second, to our knowledge, is the first example of a field theory where the vakonomic method seems to be preferable to the nonholonomic one (the opposite as in mechanics).

\section{A. A skate on an inclined plane}

This is the model of a skate (or better a knife edge, as called in Ref. 4) that moves on an inclined plane keeping the velocity of its middle point (that is also the unique point of contact with the plane, allowing for rotations) parallel to the blade (see Fig. 1).

The kinematic variables are the coordinates $(x, y)$ of the contact point and the direction $\theta$ of the blade (thus the configuration bundle $C$ is $\mathbb{R} \times \mathbb{R}^{2} \times S^{1} \rightarrow \mathbb{R}$ ). The Lagrangian is

$$
L=\frac{m}{2}\left(\dot{x}^{2}+\dot{y}^{2}\right)+\frac{I}{2} \dot{\theta}^{2}-m g_{\mathrm{eff}} y,
$$

while the constraint $S \subset J^{1} C$ is given by

$$
\dot{x} \sin \theta-\dot{y} \cos \theta=0 .
$$

\section{The nonholonomic setting}

A Chetaev admissible variation for this constraint is a vector field $W \in \Gamma(V C)$, locally identified by the three components $\left(W_{x}, W_{y}, W_{\theta}\right)$ with respect to the natural basis $(\partial / \partial x, \partial / \partial y, \partial / \partial \theta)$, that satisfies condition 3 of Definition 7: 


$$
\sin \theta W_{x}-\cos \theta W_{y}=0 .
$$

A parametrization of the admissible variations can be found by solving the previous equation as follows.

Let us introduce the subbundle $K \subset V C$ identified as the kernel of the vector bundle morphism $\left(W_{x}, W_{y}, W_{\theta}\right) \mapsto W_{x}-W_{y}$. It has a two dimensional fiber and using fiber coordinates $\left(W_{1}, W_{2}\right)$ the fibered immersion in $C$ reads as $\left(W_{1}, W_{2}\right) \mapsto\left(W_{1}, W_{1}, W_{2}\right)$. In formal language a faithful Chetaevadapted parametrization (with zero order and zero rank) is the fibered morphism

$$
\begin{gathered}
\mathbb{P}_{S}: C \rightarrow W^{*} B \otimes_{C} V C / \forall \sigma \in \Gamma(C), \quad \forall W \in \Gamma(V B), \\
\left\langle\mathbb{P}_{S} \mid\left(W_{1}, W_{2}\right)\right\rangle \circ(x(t), y(t), \theta(t))=\left(\cos \theta W_{1}, \sin \theta W_{1}, W_{2}\right) .
\end{gathered}
$$

Varying the Lagrangian along this parametrization one gets the following first variation formula:

$$
\begin{aligned}
\left\langle\delta L \mid j^{1}\left\langle\mathbb{P}_{S} \mid W\right\rangle\right\rangle \circ j^{1} \sigma=m \dot{x}\left(\cos \theta W_{1}\right)-m g_{\text {eff }}\left(\sin \theta W_{1}\right)+m \dot{y}\left(\sin \theta W_{1}\right)+I \dot{\theta} \dot{W}_{2} \\
=-m\left[\ddot{x} \cos \theta+\left(g_{\text {eff }}+\ddot{y}\right) \sin \theta\right] W_{1}-I \ddot{\theta} W_{2}+\left[m(\dot{x} \cos \theta+\dot{y} \sin \theta) W_{1}+I \dot{\theta} W_{2}\right]
\end{aligned}
$$

so that the equations of motion are

$$
\begin{gathered}
\ddot{x} \cos \theta+\left(g_{\text {eff }}+\ddot{y}\right) \sin \theta=0, \\
\ddot{\theta}=0 .
\end{gathered}
$$

Remark 1: One can check that these equations are exactly the same that can be derived from the traditional rule $\mathbb{E}_{i}=\lambda_{(\alpha)} \partial \Phi^{(\alpha)} / \partial \dot{y}^{i}$, where $\mathbb{E}_{i}$ are the Lagrange equations of the unconstrained variational problem (see Refs. 4 and 8).

\section{The vakonomic setting}

A vak-admissible variation for this constraint is a vector field $W \in \Gamma(V C)$, locally identified by the three components $\left(W_{x}, W_{y}, W_{\theta}\right)$ with respect to the natural basis $(\partial / \partial x, \partial / \partial y, \partial / \partial \theta)$, that satisfies (see Definition 14) the following condition:

$$
\sin \theta \dot{W}_{x}-\cos \theta \dot{W}_{y}+(\cos \theta \dot{x}+\sin \theta \dot{y}) W_{\theta}=0 .
$$

A parametrization of admissible variations can be found by solving the previous equation. Let us introduce the vector subbundle $K \subset V C$ identified by $W_{\theta}=0$. In formal language a faithful vakadapted parametrization (with order and rank both equal to 1) if $\dot{y} \cos \theta+\dot{x} \sin \theta \neq 0$ is the fibered morphism

$$
\begin{gathered}
P_{S}: J^{1} C \rightarrow\left(J^{1} V B\right)^{*} \otimes_{J^{1} C} V C / \forall \sigma \in \Gamma(C), \quad \forall W \in \Gamma(V B), \\
\left\langle P_{S} \mid j^{1}\left(W_{x}, W_{y}\right)\right\rangle \circ(x(t), y(t), \theta(t))=\left(W_{x}, W_{y}, \frac{\sin \theta \dot{W}_{x}-\cos \theta \dot{W}_{y}}{\dot{y} \cos \theta+\dot{x} \sin \theta}\right) .
\end{gathered}
$$

Varying the Lagrangian along the parametrization gives the following first variation formula:

$$
\left\langle\delta L \mid j^{1}\left\langle\mathbb{P}_{S} \mid W\right\rangle\right\rangle \circ j^{1} \sigma=m \dot{x} \dot{W}_{x}-m g_{\text {eff }} W_{y}+m \dot{y} \dot{W}_{y}+I \dot{\theta}\left(\frac{\sin \theta \dot{W}_{x}-\cos \theta \dot{W}_{y}}{\dot{y} \cos \theta+\dot{x} \sin \theta}\right) .
$$

Integrating by parts the derivatives of the variations leads to the following equations of motion: 


$$
\begin{gathered}
m \ddot{x}=I\left(\frac{\ddot{\theta} \sin \theta}{\dot{y} \cos \theta+\dot{x} \sin \theta}\right), \\
m \ddot{y}+m g_{\text {eff }}=-I\left(\frac{\ddot{\theta} \cos \theta}{\dot{y} \cos \theta+\dot{x} \sin \theta}\right) .
\end{gathered}
$$

Remark 2: One can check that these equations are exactly the same that can be derived from the Lagrange multiplier traditional rule (see Refs. 4 and 9): in fact, from the variation of the Lagrangian $L^{\prime}=L+\lambda_{(\alpha)} \Phi^{(\alpha)}$ one gets the following equations:

$$
\begin{gathered}
\dot{x} \sin \theta-\dot{y} \cos \theta=0, \\
m \ddot{x}=(\lambda \sin \theta), \\
m \ddot{y}+m g_{\mathrm{eff}}=-(\lambda \cos \theta), \\
I \ddot{\theta}=\lambda(\cos \theta \dot{x}+\sin \theta \dot{y}) .
\end{gathered}
$$

If $\dot{y} \cos \theta+\dot{x} \sin \theta \neq 0$ one can solve the last one for $\lambda$ and substitute in the others to get again Eq. (12).

Remark 3: Let us notice that due to the particularly simple form of the constraint equation we can solve it for $\theta$ in some open subset of the domain getting $\theta=\arctan \dot{\dot{y}}$. This also is the reason why in this case it is so easy to find a vak-adapted parametrization. If one now substitutes this expression for $\theta$ into the Lagrangian, reducing the configuration bundle but increasing the order of the Lagrangian, and then one varies it with respect to the independent variable $(x, y)$ one gets again Eq. (12).

\section{Comparison}

For a comparison of the solution we refer the reader to Ref. 4, where it has been shown that for some given trajectories the vakonomic and nonholonomic are very much different. An experimental test of the real observability of vak and $\mathrm{NH}$ trajectories in a different mechanical system has been carried out in Ref. 14 where the authors found that realistic trajectories obey NH equations.

\section{B. Relativistic hydrodynamics}

Here we present an example of a field theory where the vakonomic method and the nonholonomic one give very different result. In particular, we find that the nonholonomic theory seems to be nonphysical (every admissible section is a solution). Let us consider a region $M$ of space-time with metric $g_{\alpha \beta}$ (here we regard it as fixed, but if we want to study the coupling with gravity the formalism works as well, see Ref. 2) filled with a barotropic and isoentropic fluid. The world lines of the fluid particles and its density describe completely the configuration of the system. There are different methods to describe the kinematics. We choose to use a vector density $J^{\mu}$ physically interpreted as follows: let the unit vector field $u^{\mu}\left(g_{\alpha \beta}, J^{\mu}\right)=J^{\mu} /|J|$ be tangent to the flow lines in every point, and the scalar $\rho\left(g_{\alpha \beta}, J^{\mu}\right)=|J| / \sqrt{|g|}$ be the density of the fluid. The configuration bundle $C$ is thence that of vector densities of weight -1 [the transformation rule of $J^{\mu}$ for a coordinate change $x^{\mu^{\prime}}=x^{\mu^{\prime}}\left(x^{\mu}\right)$ with Jacobian matrix $M$ is $\left.J^{\mu^{\prime}}=M_{\mu}^{\mu^{\prime}} J^{\mu} \operatorname{det} M^{-1}\right]$. The dynamics of the system is controlled by the Lagrangian 


$$
L=-\sqrt{|g|}[\rho(1+e(\rho))] \mathrm{d} s,
$$

where the scalar $e(\rho)$ is the internal energy of the fluid. From the latter we can derive the pressure $P=\rho^{2}(\partial e / \partial \rho)$. The vector density $J^{\mu}$ cannot take arbitrary values because of the continuity equation

$$
\partial_{\mu} J^{\mu}=0
$$

that needs to hold. It acts as a nonintegrable constraint $S \subset J^{1} C$ on the derivatives of the fields.

Some further details on this physical system can be found in Refs. 11, 16, 12, and 17.

\section{The vakonomic setting}

A vak-admissible variation of the field $J$ with respect to the constraint $S$ is given by a vector field $W \in \Gamma(V C)$ represented in local coordinates as $W^{\mu}\left(\partial / \partial J^{\mu}\right)$, whose first jet is tangent to $S$, that is to say $\partial_{\mu} W^{\mu}=0$.

A vak-admissible parametrization is given by the morphism

$$
\mathbb{P}_{S}: J^{1} C \rightarrow\left(J^{1} T M\right)^{*} \otimes_{J^{1} C} V C
$$

such that $\forall \sigma \in \Gamma(C)$ and $\forall X \in \Gamma(T M)$,

$$
\left\langle\mathbb{P}_{s} \mid j^{1} X\right\rangle \circ j^{1} \sigma=£_{X} J^{\mu} \frac{\partial}{\partial J^{\mu}}=\left(\partial_{\nu} J^{\mu} X^{\nu}-J^{\nu} \partial_{\nu} X^{\mu}+J^{\mu} \partial_{\nu} X^{\nu}\right) \frac{\partial}{\partial J^{\mu}} .
$$

Varying the Lagrangian along the given parametrization gives the following expression:

$$
\left\langle\delta L \mid j^{1} X\right\rangle \circ \sigma=-\sqrt{|g|} \frac{\partial \mu}{\partial \rho} \frac{J_{\mu}}{\rho}\left(\partial_{\nu} J^{\mu} X^{\nu}-J^{\nu} \partial_{\nu} X^{\mu}+J^{\mu} \partial_{\nu} X^{\nu}\right) \mathrm{d} s,
$$

where $\mu=\rho(1+e(\rho))$ and the following identities hold:

$$
\rho \frac{\partial \mu}{\partial \rho}=\mu+P \quad \text { and } \quad \rho \mathrm{d}_{\nu}\left(\frac{\partial \mu}{\partial \rho}\right)=\mathrm{d}_{\nu} P .
$$

Integrating by parts the derivatives of $X$ one finds the following field equations:

$$
\left(u_{\mu} u^{\nu}+\delta_{\mu}^{\nu}\right) \nabla_{\nu} P+(\mu+P) u^{\nu} \nabla_{\nu} u_{\mu}^{\prime}=0 .
$$

Remark 4: In literature one can also find a different description of the system where the fundamental fields are three scalars $R^{a}\left(x^{\mu}\right)$ physically interpreted as the labels identifying the abstract fluid particle passing through the point $x^{\mu} \in M$. The quantities $J^{\mu}, u^{\mu}$, and $\rho$ are then defined as suitable functions of the fundamental fields and their first derivatives that automatically solve the constraint. This description is dynamically equivalent to our one and it performs, as in Remark 3, a reduction of the configuration bundle (three fundamental fields instead of four) increasing the order of the Lagrangian in such a way that the variations of the fundamental fields automatically preserve the constraint. We refer the reader to Refs. 17, 16, and 12 for further details.

Remark 5: The parametrization (13) we introduced is vak adapted to the constraint $\partial_{\mu} J^{\mu}$ $=0$, but not faithful to it in general. Depending on the solution sometimes it is possible to find variations of $J^{\mu}$ tangent to $S$ and vanishing on the boundary that cannot be produced by means of the parametrization (13) and a vector field $X$ that vanishes on the boundary. An example is drawn in Fig. 2. Let us consider a cylinder with the induced metric from $\mathbb{R}^{3}$. Let the configuration $\mathrm{J}^{\mu}$ be the set of unit vectors tangent to the vertical lines drawn in the left side of the figure. Let us consider a one parameter family of diffeomorphisms that realize twistings like the one drawn in the right side. They do not affect $J^{\mu}$ (nor its derivatives) on the boundary of the cylinder. Nevertheless the infinitesimal generator of the family is a vector field $X$ which does not vanish on the 

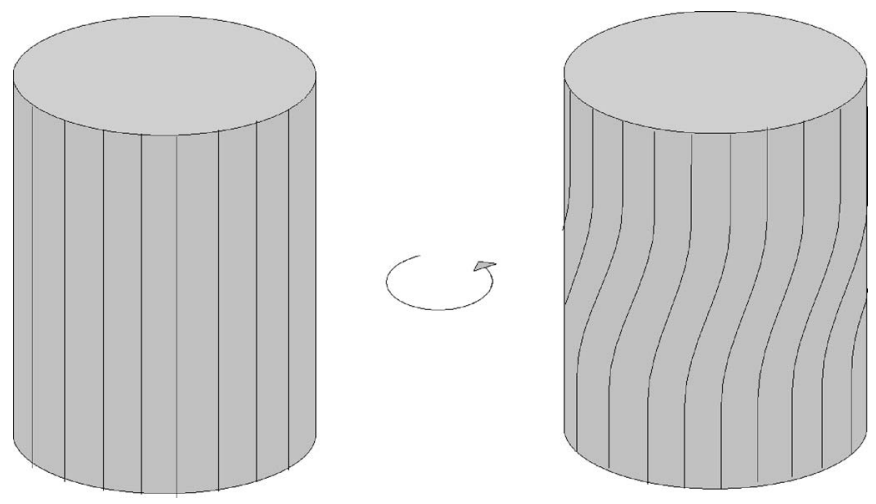

FIG. 2. Nonfaithfulness of $\mathbb{P}_{S}$.

upper part of the boundary: we have $\left.\mathcal{L}_{X} J^{\mu}\right|_{\partial D}=0$ also if $\left.X\right|_{\partial D} \neq 0$. On the other hand this is exactly what we want to do from the physical viewpoint: when we think to the congruence of curves that represent our fluid and we imagine to vary them leaving the boundary fixed, we mean that on the boundary the curves are fixed not only their tangent vectors! To support our choice to vary fields along our parametrization we stress that also in the alternative approach of abstract fluid particles variations leave unchanged the particle identification on the boundary. Solutions of the Euler-Lagrange equations (14) are not necessarily vak-critical solutions of the variational problem with the constraint $S$ given by $\partial_{\mu} J^{\mu}=0$, but anyway they represent the motions of physical fluids.

\section{The nonholonomic setting}

A Chetaev-admissible variation of the field $J$ with respect to the constraint $\Phi=\partial_{\mu} J^{\mu}=0$ is given by a vector field $W \in \Gamma(V C)$ represented in local coordinates as $W^{\mu}\left(\partial / \partial J^{\mu}\right)$ that satisfies condition 3 of Definition 7:

$$
\frac{\partial \Phi}{\partial \partial_{\alpha} J^{\mu}} W^{\mu}=0 \quad \Leftrightarrow \quad W=0 .
$$

For the relativistic fluid, thus, the unique Chetaev-admissible variation is identically vanishing. One cannot define any nontrivial variational framework with an empty set of variations and insisting on this route one obtains that any section $\sigma \in \Gamma(C)$ is Chetaev critical and thanks to Proposition 2 also $\mathbb{P}_{S}$ critical for every Chetaev-adapted parametrization. This conclusion is clearly nonphysical.

\section{CONCLUSIONS}

We have shown how the parametrized variational calculus can contribute to the study of nonholonomic and vakonomic field theories. We have defined the notion of vak criticality and Chetaev criticality and compared them with the one of criticality with respect to a vak adapted or a Chetaev-adapted parametrization. We have also shown examples in mechanics and field theory. In particular, we think that relativistic hydrodynamics is the first case where it has been shown that the vakonomic method is preferable to the nonholonomic one (the opposite result with respect to mechanics). We still cannot guess why this occurs, nor whether it is a general rule for field theories; nevertheless it seems to us that it is an interesting occurrence deserving further investigations. 
${ }^{1}$ Arnol'd, V. I., Kozlov, V. V., and Neĭshtadt, A. I., Dynamical Systems. III, Encyclopaedia of Mathematical Sciences Vol. 3 (Springer-Verlag, Berlin, 1988). Translated from the Russian by A. Iacob.

${ }^{2}$ Bibbona, E., Fatibene, L., and Francaviglia, M., "Gauge-natural parameterized variational problems, vakonomic field theories and relativistic hydrodynamics of a charged fluid," Int. J. Geom. Methods Mod. Phys. 3(8), 1573-1608 (2006).

${ }^{3}$ Binz, E., de León, M., de Diego, D. M., and Socolescu, D., "Nonholonomic constraints in classical field theories," Rep. Math. Phys. 49, 151-166 (2002); 33rd Symposium on Mathematical Physics, Torún, 2001.

${ }^{4}$ Block, A. M., Baillieul, J., Crouch, P., and Marsden, J., Nonholonomic Mechanics and Control, Interdisciplinary Applied Mathematics Vol. 24 (Springer, New York, 2003).

${ }^{5}$ Chetaev, N. G., Theoretical Mechanics (Springer-Verlag, Berlin, 1989). Translated from the Russian, lectures delivered around 1950.

${ }^{6}$ Fatibene, L. and Francaviglia, M., Natural and Gauge Natural Formalism for Classical Field Theories (Kluwer Academic, Dordrecht, 2003). A geometric perspective including spinors and gauge theories.

${ }^{7}$ Fernández, A., García, P. L., and Rodrigo, C., "Stress-energy-momentum tensors for natural constrained variational problems," J. Geom. Phys. 49, 1-20 (2004).

${ }^{8}$ García, P. L. and Rodrigo, C., "The momentum map in vakonomic mechanics," Proceedings of the XII Fall Workshop on Geometry and Physics, Publ. R. Soc. Mat. Esp. Vol. 7 (R. Soc. Mat. Esp., Madrid, 2004), pp. 111-123.

${ }^{9}$ García, P. L., García, A., and Rodrigo, C., "Cartan forms for first order constrained variational problems," J. Geom. Phys. 56, 571-610 (2006)

${ }^{10}$ Gràcia, X., Marín-Solano, J., and Muñoz-Lecanda, M.-C., "Some geometric aspects of variational calculus in constrained systems," Rep. Math. Phys. 51, 127-148 (2003).

${ }^{11}$ Hawking, S. W. and Ellis, G. F. R., The Large Scale Structure of Space-time, Cambridge Monographs on Mathematical Physics Vol. 1 (Cambridge University Press, London, 1973).

${ }^{12}$ Kijowski, J., Pawlik, D., and Tulczyjew, W., "A variational formulation of non-gravitating and gravitating hydrodynamics,” Bull. Acad. Polon. Sci. Sér. Sci. Phys. Astronom. 27(1), 163-170 (1979).

${ }^{13}$ Krupková, O. and Volný, P., "Euler-Lagrange and Hamilton equations for non-holonomic systems in field theory," J. Phys. A 38, 8715-8745 (2005).

${ }^{14}$ Lewis, A. D. and Murray, R. M., "Variational principles for constrained systems: Theory and experiment," Int. J. Non-Linear Mech. 30, 793-815 (1995).

${ }^{15}$ Marsden, J. E., Pekarsky, S., Shkoller, S., and West, M., "Variational methods, multisymplectic geometry and continuum mechanics," J. Geom. Phys. 38, 253-284 (2001).

${ }^{16}$ Soper, E., Classical Field Theory (Wiley, New York, 1976).

${ }^{17}$ Taub, A. H., "General relativistic variational principle for perfect fluids," Phys. Rev. 94, 1468-1470 (1954).

${ }^{18}$ Vankerschaver, J., Cantrijn, F., de León, M., and de Diego, D. M., "Geometric aspects of nonholonomic field theories," Rep. Math. Phys. 56, 387-411 (2005). 